\title{
CyberKnife with tumor tracking: an effective treatment for high-risk surgical patients with single peripheral lung metastases
}

\section{James W. Snider ${ }^{1}$, Eric K. Oermann ${ }^{1}$, Viola Chen ${ }^{1}$, Jennifer Rabin ${ }^{1}$, Simeng Suy ${ }^{1}, X_{i a} Y_{u}{ }^{1}$, Saloomeh Vahdat ${ }^{2}$, Sean P. Collins ${ }^{1}$, Filip Banovac ${ }^{3}$, Eric Anderson ${ }^{4}$ and Brian T. Collins ${ }^{1}{ }^{*}$}

${ }^{1}$ Department of Radiation Medicine, Georgetown University Hospital, Washington, DC, USA

2 Department of Pathology, Georgetown University Hospital, Washington, DC, USA

${ }^{3}$ Department of Radiology, Georgetown University Hospital, Washington, DC, USA

${ }^{4}$ Division of Pulmonary, Critical Care and Sleep Medicine, Georgetown University Hospital, Washington, DC, USA

Edited by:

Minesh P. Mehta, Northwestern University, USA

Reviewed by:

O. Kenneth MacDonald, Providence Medical Center, USA

Jonathan P. S. Knisely, North Shore

University Hospital, USA

\section{*Correspondence:}

Brian T. Collins, Department of

Radiation Medicine, Georgetown

University Hospital, 3800 Reservoir

Road, Northwest, Washington, DC

20007, USA.

e-mail:collinsb@

gunet.georgetown.edu
Standard treatment for operable patients with single peripheral lung metastases is metastasectomy. We report mature CyberKnife outcomes for high-risk surgical patients with biopsy proven single peripheral lung metastases. Twenty-four patients (median age 73 years) with a mean maximum tumor diameter of $2.5 \mathrm{~cm}$ (range, $0.8-4.5 \mathrm{~cm}$ ) were treated over a 6-year period extending from September 2004 to September 2010 and followed for a minimum of 1 year or until death. A mean dose of $52 \mathrm{~Gy}$ (range, 45-60 Gy) was delivered to the prescription isodose line in three fractions over a 3-11 day period (mean, 7 days). At a median follow-up of 20 months, the 2-year Kaplan-Meier local control and overall survival rates were 87 and $50 \%$, respectively. CyberKnife with fiducial tracking is an effective treatment for high-risk surgical patients with single small peripheral lung metastases. Trials comparing CyberKnife with metastasectomy for operable patients are necessary to confirm equivalence.

Keywords: lung metastases, CyberKnife, SBRT, metastasectomy

\section{INTRODUCTION}

Historically, metastatic tumors have been treated with palliative systemic and local therapies. Radiation therapy was delivered selectively in moderate doses to alleviate tumor related symptoms. This approach contrasts with current innovative treatment paradigms, which often emphasize combination therapies, where surgery and radiation therapy are designed to eradicate gross tumor and systemic therapy to eliminate micrometastases.

The lung represents a common site of metastatic disease (Pastorino, 1997). Frequently, it is the only organ involved and tumors are limited in number (Casiraghi et al., 2011). In this setting, chemotherapy and metastasectomy have been combined to enhance overall survival. The International Registry of Lung Metastases (1997) reported an increase in 5 year survival following complete metastasectomy, 36 vs. 13\% (1997). This result suggests that the complete eradication of gross lung metastases enhances overall survival. Recently, the European Institute of Oncology reported 2- and 5-year overall survival rates of 74 and $46 \%$, respectively, with modern patient selection, contemporary systemic therapy, and complete metastasectomy (Casiraghi et al., 2011).

For high-risk surgical patients and those electing not to pursue surgery, stereotactic body radiotherapy (SBRT) can reliably eradicate lung metastases (Siva et al., 2010). SBRT trials, utilizing adequate tumor doses and standard margins $(5 \mathrm{~mm}$ in the axial plane and $10 \mathrm{~mm}$ in the cranial-caudal plane), have consistently reported local control rates at 2 years of approximately $90 \%$
(Siva et al., 2010). Preliminary data suggests that CyberKnife (Accuray Incorporated, Sunnyvale, CA, USA) treatment with fiducial tracking reliably eradicates pulmonary metastases with additional lung tissue sparing (Collins et al., 2007). Continuous tracking of tumor motion due to respiration and highly accurate beam alignment throughout treatment with the CyberKnife facilitates the accurate delivery of dose distributions with tighter margins on the gross tumor volume (GTV) than historically feasible with conventional SBRT (Hoogeman et al., 2009).

We began treating high-risk surgical patients with single lung metastases in mid 2004 using the CyberKnife frameless robotic radiosurgery system with Synchrony tumor motion tracking (Collins et al., 2007). The goal of this treatment was to maintain the impressive local control rates of conventional SBRT while further decreasing radiation-induced lung damage with tight $5 \mathrm{~mm}$ margins. We report local control and overall survival rates for 24 consecutively treated, patients at high-risk for surgical management with single peripheral lung metastases treated using the CyberKnife system with fiducial tracking.

\section{MATERIALS AND METHODS ELIGIBILITY}

The Medstar Health Research Institute-Georgetown University Oncology institutional review board approved this retrospective analysis of a prospectively collected database. All participants provided informed written consent. The Georgetown University Hospital multidisciplinary thoracic oncology team evaluated 
patients. Prior to treatment, CT imaging of the chest, abdomen, and pelvis with IV contrast, PET imaging, and routine pulmonary function tests (PFTs) were completed when feasible. High-risk surgical patients with pathologically confirmed single peripheral lung metastases measuring $5 \mathrm{~cm}$ or less in maximum diameter were considered for treatment. High-risk was defined by the presence of any of the following: a post-bronchodilator percent predicted forced expiratory volume in $1 \mathrm{~s}$ (FEV1) of less than $50 \%$, a carbon monoxide diffusing capacity (DLCO) of less than $50 \%$, age greater than 75, prior lobectomy, or severe comorbid medical conditions with poor performance status. A metastasis was considered a single peripheral metastasis if there was no evidence of additional gross metastases at the time of treatment per PET/CT imaging and the lesion could be treated while respecting critical central structure radiation dose limits (Table $\mathbf{1}$ ). Finally, candidates were excluded from treatment if fiducials could not be safely placed for tumor tracking.

\section{FIDUCIAL PLACEMENT}

Under conscious sedation and with local anesthesia, three to five gold fiducials measuring $0.8-1 \mathrm{~mm}$ in diameter and $3-7 \mathrm{~mm}$ in length (Item 351-1 Best Medical International, Inc., Springfield, VA, USA) were placed for tumor tracking. CT-guidance was utilized to ensure adequate spacing $(1-2 \mathrm{~cm})$ and proximity to lesions as previously described (Yousefi et al., 2007).

\section{TREATMENT PLANNING}

Fine-cut $(1.25 \mathrm{~mm})$ treatment planning CTs were routinely obtained 7-10 days after fiducial placement during a full inhalation breath-hold. GTV were contoured utilizing lung windows. The GTV margin was expanded $5 \mathrm{~mm}$ to establish the planning treatment volume (PTV). All critical central thoracic structures (Table 1) and the lungs were contoured to ensure that incidental radiation delivered to those structures was limited. The dose to the chest wall and skin was not limited. A treatment plan was generated using the CyberKnife non-isocentric, inverseplanning ray-tracing algorithm with tissue density heterogeneity corrections for lung. Lower doses within the institutions accepted range of 45-60 Gy in three fractions were prescribed at the last author's discretion when concerns about adjacent critical structures arose (Table 1) and/or when patients were felt to have severe pulmonary dysfunction. The radiation was delivered to an isodose line that covered at least $95 \%$ of the PTV. The percentage of the total lung volume receiving $15 \mathrm{~Gy}$ or more (V15) was limited to $15 \%$.

Table 1 | Critical central structure radiation point dose limits.

\begin{tabular}{ll}
\hline Adjacent structure & Maximum dose limit (total for $\mathbf{3}$ fractions; Gy) \\
\hline Spinal cord & 18 \\
Esophagus & 27 \\
Heart & 30 \\
Main bronchus & 30 \\
Trachea & 30 \\
Great vessels & 40
\end{tabular}

\section{TREATMENT DELIVERY}

Patients were treated according to the Georgetown University Hospital small peripheral pulmonary nodule protocol as previously described (Collins et al., 2007). Briefly, patients were positioned supine and unrestrained on the treatment table with their arms at their sides, and they were placed in a tightly fitted vest with three red light emitting diodes (LEDs) on the anterior portion. The LEDs were positioned over the approximate maximum respiratory excursion point of the lower chest and upper abdomen. They were adjusted to project to a camera array within the treatment room. The automated patient positioning system was utilized to adjust the treatment table, and orthogonal X-ray images were employed to register the implanted fiducials. These images were obtained by ceiling mounted X-ray sources and corresponding floor mounted amorphous silicon image detectors.

As described in detail elsewhere (Kilby et al., 2010), immediately prior to starting treatment, Synchrony creates an adaptive correlation model between the fiducial positions as imaged by the X-ray targeting system and the LEDs as imaged by the by the camera array. The X-ray targeting system reacquired fiducials periodically throughout therapy, while the LEDs were continuously tracked. The linear accelerator was moved by the robotic arm which adjusted position in real-time to maintain alignment with the target. The patient's breathing was uninhibited during this process. Fiducials were imaged prior to every third beam in order to update the correlation model and to verify treatment positioning. If the correlation model error exceeded $3 \mathrm{~mm}$ or if fiducials were misidentified, the treatment was discontinued, and the model was recreated.

\section{FOLLOW-UP STUDIES}

Examination and CT imaging were routinely performed at 3 month follow-up intervals. PET/CT imaging was completed selectively to evaluate suspicious CT changes. Local recurrence was defined as progression within $2 \mathrm{~cm}$ of the tracked fiducials per PET/CT imaging as previously described (Vahdat et al., 2010). Other failures were considered distant. Upon failure, biopsy was required to confirm local progression.

\section{STATISTICAL ANALYSIS}

Data was analyzed and graphs were prepared with the SPSS 16.02 statistical package (IBM Corporation, Armonk, NY, USA). The follow-up duration was defined as the time from the date of completion of CyberKnife treatment to the last date of follow-up or date of death. Actuarial local control and overall survival were calculated using the Kaplan-Meier method.

\section{RESULTS}

\section{PATIENT AND TUMOR CHARACTERISTICS}

Twenty-four patients ( 15 men and 9 women) with single peripheral lung metastases and an Eastern Cooperative Oncology Group (ECOG) performance status of two or less were treated over a 6-year period extending from September 2004 to September 2010 (Table 2). The mean maximum tumor diameter was $2.5 \mathrm{~cm}$ (range, $0.8-4.5 \mathrm{~cm})$. Primaries included lung adenocarcinoma $(n=7)$, lung squamous cell carcinoma $(n=7)$, esophagus $(n=2)$, uterus $(n=2)$, ovary $(n=1)$, small bowel $(n=1)$, pancreas $(n=1)$, bladder $(n=1)$, renal $(n=1)$, and skin $(n=1)$. Ninety-two percent 
Table 2 | Patient and tumor characteristics.

\begin{tabular}{|c|c|}
\hline \multicolumn{2}{|l|}{ FEV1 (L) } \\
\hline Mean & 1.67 \\
\hline Range & $0.54-2.82$ \\
\hline \multicolumn{2}{|c|}{ PREDICTED FEV1 (\%) } \\
\hline Mean & 66 \\
\hline Range & $28-121$ \\
\hline \multicolumn{2}{|c|}{ DLCO (mL/min/mmHg) } \\
\hline Mean & 13.0 \\
\hline Range & $4.5-22.1$ \\
\hline \multicolumn{2}{|c|}{ PREDICTED DLCO (\%) } \\
\hline Mean & 62 \\
\hline Range & $20-96$ \\
\hline \multicolumn{2}{|l|}{ ECOG } \\
\hline Median & 1 \\
\hline Range & $0-2$ \\
\hline \multicolumn{2}{|c|}{ AGE (YEARS) } \\
\hline Median & 73 \\
\hline Range & $53-85$ \\
\hline \multicolumn{2}{|l|}{ SEX (\%) } \\
\hline Male & 63 \\
\hline Female & 37 \\
\hline \multicolumn{2}{|l|}{ RACE $(\%)$} \\
\hline Caucasian & 79 \\
\hline African & 17 \\
\hline Asian & 4 \\
\hline Smoker (\%) & 92 \\
\hline \multicolumn{2}{|c|}{ MAXIMUM DIAMETER (cm) } \\
\hline Mean & 2.5 \\
\hline Median & 2.5 \\
\hline Range & $0.8-4.5$ \\
\hline
\end{tabular}

of the patients were smokers; the mean post-bronchodilator percent predicted FEV1 was 66\% (range, 28-121\%). Half of the patients had prior lobectomy; two thirds of the patients were not chemotherapy candidates.

\section{TREATMENT}

Treatment plans were composed of a mean of 179 pencil beams (Table 3). A mean dose of 52 Gy (range, 45-60 Gy) was delivered to the prescription isodose line in three $1-2 \mathrm{~h}$ treatments over a 3-11 day period (mean, 7 days).

\section{COMPLICATIONS}

Four patients developed pneumothorax requiring tube thoracostomy following biopsy and fiducial placement. Subsequently, all patients completed treatment without interruption. Immediately following treatment, acute toxicity consisting of mild brief fatigue was reported in the majority of patients. During the first year transient mild-to-moderate chest wall discomfort, typically lasting several weeks, developed in seven of nine patients for lesions within $5 \mathrm{~mm}$ of the pleura. Skin toxicity, chronic chest wall pain, rib fracture, and symptomatic radiation pneumonitis ( $\geq$ Grade II) were not observed in this cohort.
Table 3 | Treatment characteristics.

\begin{tabular}{ll}
\hline & Mean (range) \\
\hline Prescribed dose (Gy) in 3 treatments & $52(45-60)$ \\
Prescription isodose line (\%) & $80(75-85)$ \\
Number of beams per treatment & $179(79-279)$ \\
Treatment course (days) & $7(3-11)$
\end{tabular}

\section{DISEASE SPREAD AND SURVIVAL}

At a median follow-up of 20 months (range, 6-80 months), 2 local failures at 11 and 18 months have been confirmed. Nineteen patients died during follow-up; 11 patient deaths were attributed to additional metastases and eight were attributed to cardiopulmonary failure. No deaths were attributed to local failure or treatment. The 2-year Kaplan-Meier local control and overall survival estimates were 87 and 50\%, respectively (Figures 1 and 2).

\section{DISCUSSION}

In mid 2004, we initiated CyberKnife treatment of high-risk surgical patients with single small $(<5 \mathrm{~cm})$ peripheral lung metastases (Collins et al., 2007). Twenty-four patients were treated in 6 years and followed for a minimum of 1 year or until death. Continuous tracking of respiratory tumor motion and highly accurate beam alignment throughout treatment with the CyberKnife prompted us to deliver dose distributions with tighter $(5 \mathrm{~mm})$ margins on the GTV than historically feasible. At a median follow-up of 20 months, the 2-year Kaplan-Meier estimated local control rate is $87 \%$. Therefore, we conclude that CyberKnife with tumor tracking is a highly effective treatment for small peripheral lung metastases. Metastasectomy and CyberKnife are reasonable treatment options for high-risk surgical patients with these tumors.

Some limitations concerning our conclusions exist. PET/CT imaging surveillance promptly identifies local recurrence following metastasectomy. However, high peritumoral lung doses often result in focal radiation-induced pneumonitis and fibrosis, hampering PET/CT recurrence assessment following irradiation (Vahdat et al., 2010). Routine biopsy was not completed in our cohort given the uncertain clinical significance of early transient elevations in tumor maximum standardized uptake value $\left(\mathrm{SUV}_{\max }\right)$ following radiation treatment and the risk associated with biopsy in these high-risk surgical patients with limited salvage treatment options. As a result, confirmation of radiographic impressions was limited to biopsy in two patients following a persistent increase in tumor $\mathrm{SUV}_{\mathrm{max}}$; biopsies were not taken to confirm the absence of disease in cases in which tumor $\mathrm{SUV}_{\text {max }}$ remained low or returned to the normal range following transient elevations. Therefore, it is possible that the actual 2-year local control rate is less than the reported $87 \%$ rate.

Overall survival was vastly inferior in this group of patients when compared to published metastasectomy cohorts (Pastorino, 1997; Casiraghi et al., 2011). At a median follow-up of 20 months, the 2-year Kaplan-Meier overall survival estimate is merely $50 \%$. We limited our study to single pulmonary metastases in an effort to simplify the analysis and to prevent inflated local control rates resulting from poor overall survival. Inadvertently, we treated a poor prognosis group of patients primarily consisting of elderly 


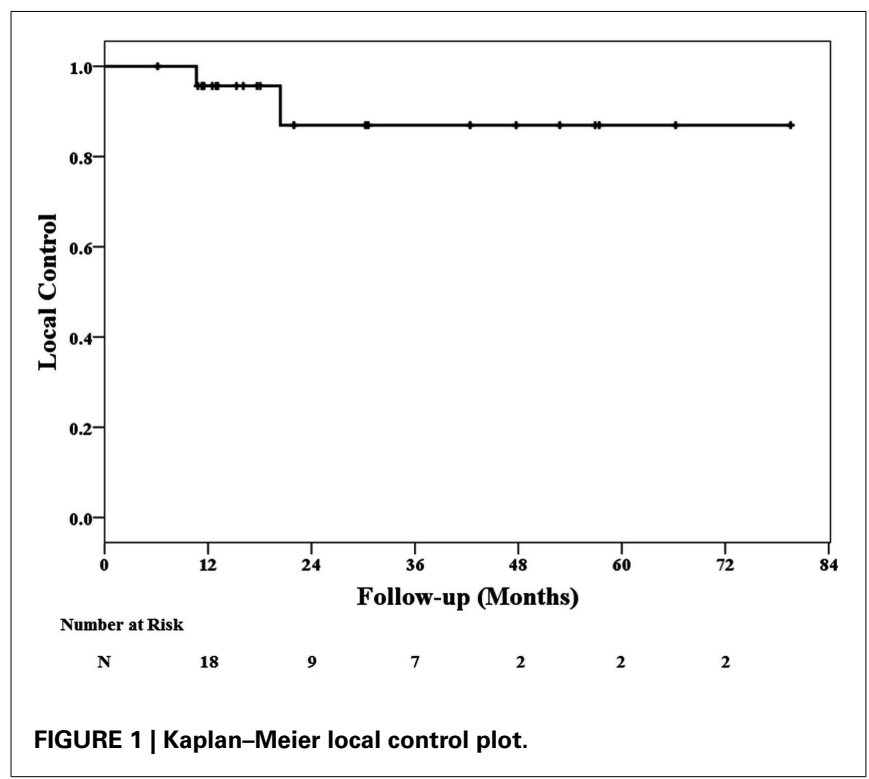

male former heavy smokers with chronic obstructive pulmonary disease (COPD) and non-small cell lung cancer (NSCLC). These patients were also generally considered poor candidates for chemotherapy. Despite the limitations of this study, we continue to treat selected consenting high-risk surgical patients with single small peripheral pulmonary metastases using CyberKnife. The encouraging local control rates in this study adequately support this practice for this frail patient population with predictably short survival (Siva et al., 2010).

Critical central structure toxicity was not observed in this trial. It is likely that toxicity was absent because we strictly adhered to conservative maximum point dose limits for critical central structures (Table 1). However, transient mild-to-moderate chest wall pain typically lasting several weeks was seen following treatment in the majority of patients with lesions within $5 \mathrm{~mm}$ of the pleura. These patients were treated conservatively with nonsteroidal anti-inflammatory medications or opioid analgesic combinations. Observations of such chest wall pain are common in lung SBRT with many studies reporting even greater amounts of significant toxicity. Indeed, rib fractures following SBRT have been reported at rates ranging from 16-23\% (Voroney et al., 2009; Dunlap et al., 2010; Nambu et al., 2011). Our treatment maintains a mean dose of $52 \mathrm{~Gy}$ which is less than standard treatment.

\section{REFERENCES}

Casiraghi, M., De Pas, T., Maisonneuve, P., Brambilla, D., Ciprandi, B., Galetta, D., Borri, A., Gasparri, R., Petrella, F., Tessitore, A., Guarize, J., Donghi, S. M., Veronesi, G., Solli, P., and Spaggiari, L. (2011). A 10-year single-center experience on 708 lung metastasectomies: the evidence of the "international registry of lung metastases.” J. Thorac. Oncol. 6, 1373-1378.

Collins, B. T., Erickson, K., Reichner, C. A., Collins, S. P., Gagnon, G. J.,
Dieterich, S., McRae, D. A., Zhang, Y., Yousefi, S., Levy, E., Chang, T., JamisDow, C., Banovac, F., and Anderson, E. D. (2007). Radical stereotactic radiosurgery with real-time tumor motion tracking in the treatment of small peripheral lung tumors. Radiat. Oncol. 2, 39.

Dunlap, N. E., Cai, J., Biedermann, G. B., Yang, W., Benedict, S. H., Sheng, K., Schefter, T. E., Kavanagh, B. D., and Larner, J. M. (2010). Chest wall volume receiving $>30$ Gy predicts risk of severe pain

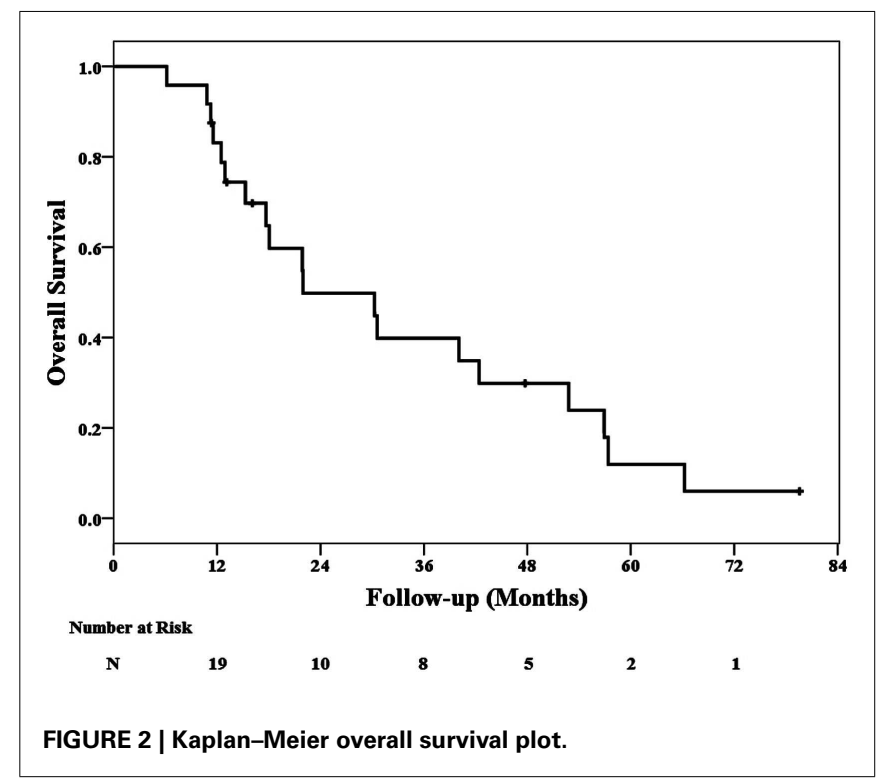

Thus, while it is tempting to further limit the dose delivered to the chest wall in these patients, this would likely result in additional local failures and is not recommended at this time given the nominal toxicity observed using our treatment approach to date

Finally, the current CyberKnife treatment approach requires the implantation of fiducials to permit tumor tracking. The procedure can result in pneumothorax, sometimes requiring tube thoracostomy, and a brief hospital stay (Collins et al., 2007). Our institution has developed a technique for placing fiducials via bronchoscopy which significantly reduces the risk of pneumothorax (Reichner et al., 2005). In our opinion, the unparalleled accuracy of fiducial tracking justifies this placement risk, which in our institution is routinely completed during required tissue confirmation of malignancy prior to radiation treatment.

\section{CONCLUSION}

CyberKnife is an effective treatment for single small peripheral lung metastases. Our experience suggests that this therapy will result in durable local control and is appropriate for highrisk surgical patients. Clinical trials comparing CyberKnife with metastasectomy for operable patients are necessary to confirm equivalence.

and/or rib fracture after lung stereotactic body radiotherapy. Int. J. Radiat. Oncol. Biol. Phys. 76, 796-801.

Hoogeman, M., Prevost, J. B., Nuyttens, J., Poll, J., Levendag, P., and Heijmen, B. (2009). Clinical accuracy of the respiratory tumor tracking system of the CyberKnife: assessment by analysis of log files. Int J. Radiat. Oncol. Biol. Phys. 74 297-303.

Kilby, W., Dooley, J. R., Kuduvalli, G., Sayeh, S., and Maurer, C.
R. Jr. (2010). The CyberKnife robotic radiosurgery system in 2010. Technol. Cancer Res. Treat. 9 , 433-452.

Nambu, A., Onishi, H., Aoki, S., Koshiishi, T., Kuriyama, K., Komiyama, T., Marino, K., Araya, M., Saito, R., Tominaga, L., Maehata, Y., Sawada, E., and Araki, T. (2011). Rib fracture after stereotactic radiotherapy on follow-up thin-section computed tomography in 177 primary lung cancer patients. Radiat. Oncol. 6, 137. 
Pastorino, U. (1997). Lung metastasectomy: why, when, how. Crit. Rev. Oncol. Hematol. 26, 137-145.

Reichner, C., Collins, B., Gagnon, G., Shakun, M., Jamis-Dow, C., and Anderson, E. (2005). The placement of gold fiducials for CyberKnife stereotactic radiosurgery using a modified transbronchial needle aspiration technique. J. Bronchol. 12, 193-195.

Siva, S., MacManus, M., and Ball, D. (2010). Stereotactic radiotherapy for pulmonary oligometastases: a systematic review. J. Thorac. Oncol. 5, 1091-1099.

The International Registry of Lung Metastases. (1997). Long-term results of lung metastasectomy: prognostic analyses based on 5206 cases. J. Thorac. Cardiovasc. Surg. 113, 37-49.
Vahdat, S., Oermann, E. K., Collins, S. P., Yu, X., Abedalthagafi, M. Debrito, P., Suy, S., Yousefi, S., Gutierrez, C. J., Chang, T., Banovac, F., Anderson, E. D., Esposito, G., and Collins, B. T. (2010). CyberKnife radiosurgery for inoperable stage IA non-small cell lung cancer: 18F-fluorodeoxyglucose positron emission tomography/computed tomography serial tumor response assessment. J. Hematol. Oncol. 3,6 .

Voroney, J. P., Hope, A., Dahele, M. R., Purdie, T. G., Franks, K. N., Pearson, S., Cho, J. B., Sun, A., Payne, D. G., Bissonnette, J. P., Bezjak, A., and Brade, A. M. (2009). Chest wall pain and rib fracture after stereotactic radiotherapy for peripheral nonsmall cell lung cancer. J. Thorac. Oncol. 4, 1035-1037.
Yousefi, S., Collins, B. T., Reichner, C. A., Anderson, E. D., Jamis-Dow, C., Gagnon, G., Malik, S., Marshall, B., Chang, T., and Banovac, F. (2007). Complications of thoracic computed tomography-guided fiducial placement for the purpose of stereotactic body radiation therapy. Clin. Lung Cancer 8, 252-256.

Conflict of Interest Statement: The authors declare that the research was conducted in the absence of any commercial or financial relationships that could be construed as a potential conflict of interest.

Received: 16 December 2011; accepted: 31 May 2012; published online: 29 June 2012.
Citation: Snider JW, Oermann EK, Chen $V$, Rabin J, Suy $S, Y u \quad X$, Vahdat S, Collins SP, Banovac $F$, Anderson E and Collins BT (2012) CyberKnife with tumor tracking: an effective treatment for high-risk surgical patients with single peripheral lung metastases. Front. Oncol. 2:63. doi 10.3389/fonc. 2012.00063

This article was submitted to Frontiers in Radiation Oncology, a specialty of Frontiers in Oncology.

Copyright (c) 2012 Snider, Oermann, Chen, Rabin, Suy, Yu, Vahdat, Collins, Banovac, Anderson and Collins. This is an open-access article distributed under the terms of the Creative Commons Attribution Non Commercial License, which permits non-commercial use, distribution, and reproduction in other forums, provided the original authors and source are credited. 